\title{
Guest editorial—special issue on cloud computing
}

\author{
Dilma Da Silva $\cdot$ Dongyan Xu $\cdot$ Dan Reed
}

Published online: 25 August 2011

(C) The Brazilian Computer Society 2011

Cloud Computing has been receiving a lot of attention from both the academic community and the computing industry. As recently as four years ago, it was easy to find people who perceived cloud computing as the "buzzword of the day", the latest computing fad soon to be replaced by a new idea promising to revolutionize the information world. Nowadays one can still find a lot of excitement and some hype around the term, but the discourse has shifted. There is a general acknowledgement that this new model for delivery of computing services has already had lasting impact in our industry and that it will continue to influence how computing resources are accessed and managed, how datacenters are built, and how business and economic models for computing services evolve.

Much of the basis for Cloud Computing comes from a collection of many old and few new concepts in several research fields like Service-Oriented Architectures (SOA), virtualization, and distributed and grid computing. Cloud Computing introduces challenges and new possibilities in many aspects of computing, including Internet architecture, protocols, services, and applications. The computing community is in the process of refining and reframing problems and ap-

D. Da Silva $(\bowtie)$

IBM TJ Watson Research Center, New York, USA

e-mail:dilmasilva@us.ibm.com

D. Xu

Department of Computer Science and Department of Electrical and Computing Engineering, Purdue University, West Lafayette, USA

e-mail: dxu@cs.purdue.edu

\section{Reed}

Technology Policy and Strategy and leader of the eXtreme Computing Group at Microsoft Research, Redmond, USA e-mail: daniel.Reed@microsoft.com proaches for the basis of cloud computing. The large number of papers recently published in the topic documents the collective ongoing effort of establishing the basis for the work in the field, with very few final answers available. In this context, this Special Issue contributes to the Cloud Computing dialogue by highlighting novel exciting approaches for resource management.

The selected papers of our Special Issue in Cloud Computing will appear in two parts. The following paragraphs provide a brief description of the three papers in this issue.

In "Autonomic live adaptation of virtual networked environments in a multi-domain infrastructure", Ruth, Ree, Xu, and Goasguen address the issue of how to federate multiple cloud domains. They propose to extend the usual view of machine and network virtualization to create virtual computing environments that are able to automatically relocate themselves across infrastructure boundaries.

Borger, Rosseto, Parra, and Dantas focus on the interaction between mobile and grid environments in their paper "MITSIO - an architecture for the management of interactive tasks and the semantic integration of ontologies in the mobile grid". The paper introduces an architecture for transparent provisioning of grid or cloud computing resources for mobile users. The authors provide evidence that the architecture is provider-agnostic, allowing for selection of resources from multiple organizations. The paper also presents data on battery power savings achieved by moving computing from the mobile device to the cloud or grid.

The paper "A2HA-automatic and adaptive host allocation in utility computing for bag-of-tasks", by Ferreira, Silva, and Veiga, proposes an approach for running the Bagof-Tasks class of parallel applications in the cloud. The challenge in scheduling this class of applications is to determine the appropriate number of hosts without relying on information about total job processing time or task completion time. 
The paper presents an algorithm that dynamically predicts the number of cloud instances to be allocated, so that the maximum speedup can be obtained while respecting a given pre-defined expense budget.

Stay tuned for additional cloud computing papers to appear in the next JISA issue.

We would like to thank the authors for their contributions and the anonymous reviewers for their expert guidance in improving the papers. We are grateful to JISA's Editors-inChief and their associated editorial team for their excellent support.

Sincerely, Dilma Da Silva, Dongyan Xu, and Dan Reed Guest Editors 